INPLASY

PROTOCOL

To cite: Yan et al. Massage as a Safe and Effective Alternative Treatment of diabetic gastroparesis: A Systematic Review and Meta-Analysis. Inplasy protocol 202140109. doi:

10.37766/inplasy2021.4.0109

Received: 20 April 2021

Published: 21 April 2021

Corresponding author:

Yan Huixin

doctoryhx@126.com

Author Affiliation:

Yueyang Hospital of Integrated

Traditional Chinese and

Western Medicine,Shanghai

University of Traditional

Chinese Medicine

Support: SMC2013.

Review Stage at time of this submission: Preliminary searches.

Conflicts of interest:

None declared.

\section{Massage as a Safe and Effective Alternative Treatment of diabetic gastroparesis: A Systematic Review and Meta-Analysis}

Yan, HX1; An, Y2; Zhang, T3; Chu, YZ4; Zhao, JN5; Yan, JT6.

Review question / Objective: The purpose of this study is to investigate the therapeutic effect of massage on diabetic gastroparesis. According to the PICOS framework, patients with diabetic gastroparesis were selected as the research object, massage was selected as intervention measures, Western medicine conventional treatment was selected as control measures, total clinical effectiveness was selected as the main outcome indicator, and the research design was RCT trial.

Condition being studied: Diabetic gastroparesis.

Information sources: CNKI; WF; VIP; CBM; Pubmed; Embase; Cochrane; Web of Science; Medline.

INPLASY registration number: This protocol was registered with the International Platform of Registered Systematic Review and Meta-Analysis Protocols (INPLASY) on 21 April 2021 and was last updated on 21 April 2021 (registration number INPLASY202140109).

\section{INTRODUCTION}

Review question / Objective: The purpose of this study is to investigate the therapeutic effect of massage on diabetic gastroparesis. According to the PICOS framework, patients with diabetic gastroparesis were selected as the research object, massage was selected as intervention measures, Western medicine conventional treatment was selected as control measures, total clinical 
effectiveness was selected as the main outcome indicator, and the research design was RCT trial.

Condition being studied: Diabetic gastroparesis.

\section{METHODS}

Participant or population: Patients with diabetic gastroparesis.

Intervention: Massage.

Comparator: Simple western medicine routine treatment.

Study designs to be included: Clinical RCT trial.

Information sources: CNKI; WF; VIP; CBM; Pubmed; Embase; Cochrane; Web of Science; Medline.

Main outcome(s): Total clinical effectiveness.

Quality assessment / Risk of bias analysis: We will assess the following areas of the studies: sequence generation, allocation concealment, blinding of participants and assessors, blinding of outcome assessment, incomplete outcome data, selective outcome reporting, and other sources of bias. The risk of bias will be categorized into 3 levels: low risk, high risk, and unclear. If the items are unclear or insufficient, we will contrive to contact the author for further information. Any disagreements will be resolved through the discussion with the third reviewer.

Strategy of data synthesis: Data analysis and quantitative data synthesis will be conducted with RevMan V.5.3. If no substantial statistical heterogeneity is detected, fixed-effect model will be applied for data synthesis. If we detect substantial statistical heterogeneity, we will use random-effects model, and explore the possible cause from a clinical and methodological perspective with providing a descriptive or subgroup analysis.
Subgroup analysis: In order to explain the heterogeneity, a subgroup analysis will be conducted based on potential factors, including different massage techniques, treatment sites, intervention time and frequency, etc.

Sensitivity analysis: In order to verify the robustness of the review conclusions, sensitivity analyses will be conducted for the primary outcomes. The impacts of following factors will be assessed, including sample size, study design, methodological quality and missing data. After low quality study is excluded, the analysis will be repeated.

Country(ies) involved: China.

Keywords: Diabetic gastroparesis.

Contributions of each author:

Author 1 - Yan Huixin.

Email: doctoryhx@126.com

Author 2 - An Yun.

Email: anyun920122@163.com

Author 3 - Zhang Tao.

Email: 510444994@qq.com

Author 4 - Chu Yuzhou.

Email: drchuyuzhou@126.com

Author 5 - Zhao Jiangna.

Author 6 - Yan Juntao.

Email: doctoryjt@163.com 